\title{
When Women Come First
}


This page intentionally left blank 


\section{When Women Come First}

Gender and Class in

Transnational Migration

Sheba Mariam George 
University of California Press

Berkeley and Los Angeles, California

University of California Press, Ltd.

London, England

(C) 2005 by The Regents of the University of California

Library of Congress Cataloging-in-Publication Data

George, Sheba Mariam, 1966-.

When women come first : gender and class in transnational migration / Sheba Mariam George.

p. $\mathrm{cm}$.

Includes bibliographical references and index.

ISBN 0-520-243I8-8 (cloth : alk. paper) - ISBN 0-520-24319-6 (pbk. : alk. paper)

I. Women, East Indian - United States - Social conditions. 2. Women immigrants - United States - Social conditions. 3. Women, East Indian Employment - United States. 4. Women immigrants - Employment Social aspects - United States. 5. Nurses - United States - Social conditions. 6. East Indians - United States - Social conditions.

7. Sex role-United States. 8. Man-woman relationships - United States. 9. Man-woman relationships - India. Io. Transnationalism. I. Title.

EI $84 . \mathrm{E} 2 \mathrm{G} 46 \quad 2005$

$305.48^{\prime} 8914 \mathrm{IIO} 73-\mathrm{dc2} 2$

2004020977

Manufactured in the United States of America
$\begin{array}{llllllllll}\text { I4 } & \text { I3 } & \text { I2 } & \text { II } & \text { IO } & 09 & 08 & 07 & 06 & 05\end{array}$
IO $\quad 9 \begin{array}{lllllllll} & 9 & 7 & 6 & 5 & 4 & 3 & 2 & \text { I }\end{array}$

Printed on Ecobook 50 containing a minimum 50\% post-consumer waste, processed chlorine free. The balance contains virgin pulp, including $25 \%$ Forest Stewardship Council Certified for no old-growth tree cutting, processed either TCF or ECF. The sheet is acid-free and meets the minimum requirements of ANSI/NISO Z39.48-1992 (R 1997) (Permanence of Paper). 
For my parents, George Mathew and Annamma George 
This page intentionally left blank 